Appl. Math. Lett., 25, (2012), 2009-2014. 


\title{
DSM for general nonlinear equations
}

\author{
A.G. Ramm \\ Mathematics Department, Kansas State University, \\ Manhattan, KS 66506-2602, USA \\ ramm@math.ksu.edu
}

\begin{abstract}
If $F: H \rightarrow H$ is a map in a Hilbert space $H, F \in C_{l o c}^{2}$, and there exists a solution $y$, possibly non-unique, such that $F(y)=0, F^{\prime}(y) \neq 0$, then equation $F(u)=0$ can be solved by a DSM (Dynamical Systems Method) and the rate of convergence of the DSM is given provided that a source-type assumption holds. A discrete version of the DSM yields also a convergent iterative method for finding $y$. This method converges at the rate of a geometric series. Stable approximation to a solution of the equation $F(u)=f$ is constructed by a DSM when $f$ is unknown but the noisy data $f_{\delta}$ are known, where $\left\|f_{\delta}-f\right\| \leq \delta$.
\end{abstract}

\section{Introduction}

In this paper a method for solving a general class of nonlinear operator equations $F(u)=0$ in a Hilbert space is proposed, its convergence is proved, and an iterative method for solving the above equation is constructed. Convergence of the iterative method is proved. These results are based on the following assumptions: a) the above equation has a solution $y$, possibly non-unique, b) $F \in C_{\text {loc }}^{2}$, and c) $F^{\prime}(y) \neq 0$. The last condition means that there exists a $z$ such that $F^{\prime}(y) z \neq 0$. This is a very weak assumption. It allows the null-space of the operator $F^{\prime}(y)$ to be infinite-dimensional. No restrictions on the rate of growth of nonlinearity are made. The literature on the methods for solving nonlinear equations is large (see, e.g., [2] and references therein). Most of the known results are based on Newtontype methods and their modifications. There is a well-developed theory for equations with monotone operators and more general classes of operator equations ([4], [5]). The method used in this paper is a version of the Dynamical Systems Method (DSM). The general development of the DSM is presented in [3]- [8]. In this paper the ideas from [6] are used. The general idea of the DSM is described briefly below. In [1] a Newton-type DSM version is proposed under the assumption that the Fréchet derivative $F^{\prime}$ is a boundedly invertible linear operator. Under this assumption many classical numerical methods for

MSC: 47H15, 47H20, 65H10, 65J15, 65N20

key words: nonlinear operator equations, iterative methods, DSM-Dynamical Systems Method 
solving operator equations $F(u)=0$ are shown in [4] to be various versions of the DSM. These methods include Newton-type, modified Newton-type, Gauss-Newton-type, gradient method, simple iterations method, etc. But the DSM allows one to treat the problems in which $F^{\prime}$ is not boundedly invertible. In [5] the numerical efficiency of the DSM is demonstrated by many examples.

Let $F: H \rightarrow H$ be a map in a Hilbert space. One can also consider the case when $F: H_{1} \rightarrow H_{2}$, where $H_{1}$ and $H_{2}$ are Hilbert spaces, but for simplicity of notations we restrict the presentation in this paper to the case of one Hilbert space $H_{1}=H_{2}=H$. The results and proofs can be rewritten for the case when $F: H_{1} \rightarrow H_{2}$. Assume that equation

$$
F(u)=0
$$

has a solution $y$, possibly non-unique, and

$$
F^{\prime}(y) \neq 0
$$

where $F^{\prime}$ is the Frèchet derivative of $F$. This assumption means that $F^{\prime}(y)$ is not equal to zero identically on $H$. Assume that $F \in C_{l o c}^{2}$, i.e.,

$$
\sup _{u \in B\left(u_{0}, R\right)}\left\|F^{(j)}(u)\right\| \leq M_{j}(R) \quad 0 \leq j \leq 2,
$$

where $u_{0} \in H$ is a given element, $R>0$, and no restrictions on the growth of $M_{j}(R)$ as $R$ grows are made. This means that the nonlinearity $F$ can grow arbitrarily fast as $\left\|u-u_{0}\right\|$ grows. Under these assumptions equation (1) may have no solutions. Thus, we have assumed that a solution $y$ to (1) exists. There are many results giving sufficient conditions for the existence of a solution to nonlinear equations, but we do not go into detail since it is not the topic of our paper.

We do not assume that $F^{\prime}(u)$ has a bounded inverse operator, so the standard Newtontype methods are not applicable. The Dynamical Systems Method (DSM) consists of finding an operator $\Phi$ such that the problem

$$
\dot{u}=\Phi(t, u), \quad u(0)=u_{0}, \quad t \geq 0, \quad \dot{u}=\frac{d u}{d t},
$$

has a unique global solution $u(t)$, (that is, the solution exists for all $t \geq 0$ ), there exists $u(\infty):=\lim _{t \rightarrow \infty} u(t)$, and $F(u(\infty))=0$. To ensure the unique local solvability of (4) we assume that

$$
\|\Phi(t, u)-\Phi(t, v)\| \leq L(R)\|u-v\| \quad \forall u, v \in B\left(u_{0}, R\right) .
$$

Then the global existence of the unique local solution holds if $\sup _{t \geq 0}\|u(t)\|<\infty$.

The results of this paper are summarized in several theorems. Let us denote

$$
A:=F^{\prime}(u(t)), T:=A^{*} A, \quad T_{a}:=T+a I ; \quad \widetilde{A}:=F^{\prime}(y), \quad \widetilde{T}=\widetilde{A}^{*} \widetilde{A} .
$$

Assume that $a(t)$ is a positive monotonically decaying function,

$$
a(t)>0, \quad \lim _{t \rightarrow \infty} a(t)=0, \quad \dot{a}<0, \quad \frac{|\dot{a}|}{a} \leq 0.4 .
$$


Theorem 1. Assume that a solution to equation (1) exists, (possibly non-unique), that assumptions (2) and (3) hold, and that

$$
y-u_{0}=\widetilde{T} v, \quad\|v\| \leq\left(20 M_{1} M_{2}\right)^{-1}, \quad\left\|y-u_{0}\right\| \leq 5 M_{1} M_{2}^{-1} .
$$

Let $a(0)=\left(5 M_{1}\right)^{2 / 3} M_{2}^{4 / 3}\left\|y-u_{0}\right\|^{4 / 3}$. Then there exists $\lim _{t \rightarrow \infty} u(t):=u(\infty):=y, F(y)=0$, and

$$
\|u(t)-y\| \leq C_{I} a^{1 / 2}(t), \quad C_{I}:=\frac{\left\|y-u_{0}\right\|^{1 / 3}}{\left(5 M_{1} M_{2}^{2}\right)^{1 / 3}},
$$

where $u(t)$ solves the DSM Cauchy problem:

$$
\dot{u}=-T_{a(t)}^{-1}\left[A^{*} F(u(t))+a(t)(u(t)-u(0))\right], \quad u(0)=u_{0},
$$

the solution of which exists globally and is unique.

Theorem 2. Under the assumptions of Theorem 1, the iterative process

$$
u_{n+1}=u_{n}-h_{n} T_{a_{n}}^{-1}\left[A^{*}\left(u_{n}\right) F\left(u_{n}\right)+a_{n}\left(u_{n}-u(0)\right)\right], \quad u_{0}=u(0),
$$

where $h_{n}>0$ and $a_{n}>0$ are suitably chosen, generates the sequence $u_{n}$ converging to $y$.

Remark 1. The suitable choices of $a_{n}$ and $h_{n}$ are made in the proof of Theorem 2.

Remark 2. Theorem 1 says that any solvable operator equation with $C_{l o c}^{2}$ operator, satisfying weak assumptions, stated in Theorem 1, can be solved by the DSM (9). Condition (2) means that the range of the linear operator $F^{\prime}(y)$ contains at least one non-zero element. This condition allows $F^{\prime}(y)$ to have an infinite-dimensional null-space.

In Section 2 we prove Theorems 1 and 2. In Section 3 and Section 4 we study the stability of the solution. In the proofs we use the following lemmas.

Lemma 1. Assume that $g(t) \geq 0$ is a $C_{\text {loc }}^{1}([0, \infty))$ function satisfying the inequality

$$
\dot{g}(t) \leq-\gamma(t) g+\alpha(t) g^{2}+\beta(t), \quad t \geq 0, \quad \dot{g}:=\frac{d g}{d t},
$$

where $\gamma(t), \alpha(t)$ and $\beta(t)$ are nonnegative continuous functions defined on $[0, \infty)$. Assume that there exists $\mu \in C^{1}([0, \infty))$, $\mu>0$, such that

$$
\beta(t)+\alpha(t) \mu^{-2} \leq \frac{1}{\mu(t)}\left(\gamma(t)-\frac{\dot{\mu}(t)}{\mu(t)}\right), \quad g(0) \mu(0) \leq 1 .
$$

Then any non-negative solution $g(t)$ to (11) exists globally, that is, on $[0, \infty)$, and

$$
0 \leq g(t) \leq \frac{1}{\mu(t)}, \quad t \in[0, \infty)
$$

A generalized version of Lemma 1 is proved in [7]. 
Lemma 2. Let $g_{n+1} \leq \gamma g_{n}+p g_{n}^{2}, g_{0}:=m>0,0<\gamma<1, p>0$. If $m<\frac{q-\gamma}{p}$, where $\gamma<q<1$, then $\lim _{n \rightarrow \infty} g_{n}=0$, and $g_{n} \leq g_{0} q^{n}$.

Proof of Lemma 2. Estimate $g_{1} \leq \gamma m+p m^{2} \leq q m$ holds if $m \leq \frac{q-\gamma}{p}, \gamma<q<1$. Assume that $g_{n} \leq g_{0} q^{n}$. Then

$$
g_{n+1} \leq \gamma g_{0} q^{n}+p\left(g_{0} q^{n}\right)^{2}=g_{0} q^{n}\left(\gamma+p g_{0} q^{n}\right)<g_{0} q^{n+1}
$$

because $\gamma+p g_{0} q^{n}<\gamma+p g_{0} q \leq \gamma+p m \leq q$. Lemma 2 is proved.

The following lemma is borrowed from [7].

Lemma 3. Assume that $g_{n} \geq 0, \alpha\left(n, g_{n}\right) \geq 0$,

$$
g_{n+1} \leq\left(1-h_{n} \gamma_{n}\right) g_{n}+h_{n} \alpha\left(n, g_{n}\right)+h_{n} \beta_{n} ; \quad h_{n}>0, \quad 0<h_{n} \gamma_{n}<1
$$

and $\alpha\left(n, g_{n}\right) \geq \alpha\left(n, p_{n}\right)$ if $g_{n} \geq p_{n}$. If there exists a sequence $\mu_{n}>0$ such that

$$
\alpha\left(n, \frac{1}{\mu_{n}}\right)+\beta_{n} \leq \frac{1}{\mu_{n}}\left(\gamma_{n}-\frac{\mu_{n+1}-\mu_{n}}{h_{n} \mu_{n}}\right),
$$

and

$$
g_{0} \leq \frac{1}{\mu_{0}}
$$

then

$$
0 \leq g_{n} \leq \frac{1}{\mu_{n}}, \quad \forall n \geq 0 .
$$

Proof. For $n=0$ inequality (17) holds because of (16). Assume that it holds for all $n \leq m$ and let us check that then it holds for $n=m+1$. If this is done, the lemma is proved. Using the inductive assumption, one gets:

$$
g_{m+1} \leq\left(1-h_{m} \gamma_{m}\right) \frac{1}{\mu_{m}}+h_{m} \alpha\left(m, \frac{1}{\mu_{m}}\right)+h_{m} \beta_{m}
$$

This and inequality (15) imply:

$$
\begin{aligned}
g_{m+1} & \leq\left(1-h_{m} \gamma_{m}\right) \frac{1}{\mu_{m}}+h_{m} \frac{1}{\mu_{m}}\left(\gamma_{m}-\frac{\mu_{m+1}-\mu_{m}}{h_{m} \mu_{m}}\right) \\
& =\mu_{m}^{-1}-\frac{\mu_{m+1}-\mu_{m}}{\mu_{m}^{2}} \leq \mu_{m+1}^{-1} .
\end{aligned}
$$

The last inequality is obvious since it can be written as $-\left(\mu_{m}-\mu_{m+1}\right)^{2} \leq 0$. Lemma 3 is proved. 


\section{Proofs of Theorems 1 and 2}

Proof of Theorem 1. Since $\widetilde{T} \neq 0$, for a suitable choice of $u_{0}$ there exists a $v$ such that $y-u(0)=\widetilde{T} v$, and we assume that $\|v\| \leq c_{1}:=\left(20 M_{1} M_{2}\right)^{-1}$. Let

$$
u(t)-y:=w(t), \quad\|w(t)\|:=g(t) .
$$

Write equation (9) as

$$
\dot{w}=-T_{a(t)}^{-1}\left[A^{*}(F(u)-F(y))+a(t) w+a(t)(y-u(0))\right],
$$

and use the formula $F(u)-F(y)=A w+K$, where $\|K\| \leq \frac{M_{2} g^{2}}{2}$. Then (18) yields

$$
\dot{w}=-w-T_{a(t)}^{-1} A^{*} K-a(t) T_{a(t)}^{-1} \widetilde{T} v .
$$

Multiply this equation by $w$ in $H$ and use the estimate $\left\|T_{a}^{-1} A^{*}\right\| \leq \frac{1}{2 \sqrt{a}}, a>0$, which follows from the spectral theorem (see [4]), to get

$$
g \dot{g} \leq-g^{2}+\frac{1}{2 \sqrt{a(t)}} \frac{M_{2} g^{3}}{2}+a(t)\left\|\left(T_{a(t)}^{-1}-\widetilde{T}_{a(t)}^{-1}+\widetilde{T}_{a(t)}^{-1}\right) \widetilde{T}\right\|\|v\| g .
$$

If $a>0$ then, by the spectral theorem, $\left\|\left(\widetilde{T}_{a}\right)^{-1} \widetilde{T}\right\| \leq 1, a\left\|T_{a}^{-1}\right\| \leq 1$, and

$$
a\left\|\left(T_{a}^{-1}-\widetilde{T}_{a}^{-1}\right) \widetilde{T}\right\|=a\left\|T_{a}^{-1}\left(A^{*} A-\widetilde{A}^{*} \widetilde{A}\right) \widetilde{T}_{a}^{-1} \widetilde{T}\right\| \leq 2 M_{1} M_{2} g .
$$

Here we have used estimate (3) and the estimate of the type $\left\|A^{*}[A(u)-\widetilde{A}(y)]\right\| \leq M_{1} M_{2} g$. Collecting the above estimates and the estimate $\|v\| \leq c_{1}$, one gets

$$
\dot{g} \leq-\frac{9 g}{10}+\frac{c_{0} g^{2}}{\sqrt{a(t)}}+c_{1} a(t), \quad c_{0}:=\frac{M_{2}}{4}, \quad c_{1}:=\left(20 M_{1} M_{2}\right)^{-1} .
$$

Apply Lemma 1 to $(20)$. Here $\gamma=\frac{9}{10}, \alpha(t)=\frac{c_{0}}{\sqrt{a(t)}}, \beta(t)=c_{1} a(t)$. Choose

$$
\mu(t)=\frac{\lambda}{\sqrt{a(t)}}, \quad \lambda=\text { const }>0, \quad \frac{\dot{\mu}}{\mu}=0.5 \frac{|\dot{a}(t)|}{a(t)} \leq 0.4 .
$$

Conditions of Lemma 1 hold if

$$
\lambda a^{-0.5}(t)\left[c_{0} a^{0.5}(t) \lambda^{-2}+c_{1} a(t)\right] \leq(0.9-0.4)=0.5,
$$

and $g(0) \lambda \leq a^{0.5}(0)$. Choose $\lambda=a^{0.5}(0) / g(0)$. Then (21) holds if

$$
c_{0} \lambda^{-1}+c_{1} a^{0.5}(0) \lambda \leq 0.5
$$

Consider the problem

$$
m(s):=c_{0} s^{-1}+c_{1} a^{0.5}(0) s=\min :=m,
$$


where the minimization is over $s>0$. The minimum is attained at $s=s_{m}:=\left(\frac{c_{0}}{c_{1}}\right)^{0.5} a(0)^{-0.25}$ and $m=2\left(c_{0} c_{1}\right)^{1 / 2} a^{1 / 4}(0)$. Note that $2\left(c_{0} c_{1}\right)^{1 / 2}=\left(20 M_{1}\right)^{-0.5}$. Thus, if $\lambda=a^{0.5}(0) / g(0)$ and $2\left(c_{0} c_{1}\right)^{1 / 2} a^{1 / 4}(0) \leq 0.5$, that is, $a(0) \leq 25 M_{1}^{2}$, then, by Lemma 1 , the solution to (9) exists for all $t \geq 0$ and

$$
\|u(t)-y\| \leq \frac{\sqrt{a(t)}\|u(0)-y\|}{a^{0.5}(0)} .
$$

In the proof of Theorem 1 we satisfied inequality (22) by taking $a(0) \leq 25 M_{1}^{2}$ and $\lambda=$ $a^{1 / 2}(0) g^{-1}(0)=\left(\frac{c_{0}}{c_{1}}\right)^{0.5} a(0)^{-0.25}$. The last relation implies $g(0)=a^{3 / 4}(0)\left(5 M_{1} M_{2}^{2}\right)^{-0.5}$. Therefore, $a^{0.5}(0)=g^{2 / 3}(0)\left(5 M_{1} M_{2}^{2}\right)^{1 / 3}$. Consequently, the right-hand side of the estimate (23) is $C_{I} a^{1 / 2}(t)$, where $C_{I}$ is defined in (8). Since $a(0) \leq 25 M_{1}^{2}$, it follows that $g(0) \leq$ $5 M_{1} M_{2}^{-1}$. Thus, the initial approximation $u_{0}$ should be not too far from the solution $y$, namely, $\left\|y-u_{0}\right\| \leq 5 M_{1} M_{2}^{-1}$, as in $(7)$.

Theorem 1 is proved.

Proof of Theorem 2. Let $w_{n}:=u_{n}-y, g_{n}:=\left\|w_{n}\right\|$. We assume that $2 M_{1} M_{2}\|v\| \leq \frac{1}{2}$ and rewrite (10) as

$$
w_{n+1}=w_{n}-h_{n} T_{a_{n}}^{-1}\left[A^{*}\left(u_{n}\right)\left(F\left(u_{n}\right)-F(y)\right)+a_{n} w_{n}+a_{n}\left(y-u_{0}\right)\right], \quad w_{0}=\left\|u_{0}-y\right\| .
$$

Using the Taylor formula $F\left(u_{n}\right)-F(y)=A\left(u_{n}\right) w_{n}+K\left(w_{n}\right), \quad\|K\| \leq \frac{M_{2} g_{n}^{2}}{2}$, the estimate $\left\|T_{a_{n}}^{-1} A^{*}\left(u_{n}\right)\right\| \leq \frac{1}{2 \sqrt{a_{n}}}$, and the formula $y-u_{0}=\widetilde{T} v$, we get

$$
w_{n+1}=\left(1-h_{n}\right) w_{n}-h_{n} T_{a_{n}}^{-1} A^{*}\left(u_{n}\right) K\left(w_{n}\right)-h_{n} a_{n} T_{a_{n}}^{-1} \widetilde{T} v
$$

Taking into account that $\left\|\widetilde{T}_{a}^{-1} \widetilde{T}\right\| \leq 1$, and $a\left\|T_{a}^{-1}\right\| \leq 1$ if $a>0$, we obtain $\left\|T_{a_{n}}^{-1} \widetilde{T} v\right\| \leq$ $\left\|\left(T_{a_{n}}^{-1}-\widetilde{T}_{a_{n}}^{-1}\right) \widetilde{T}\right\|\|v\|+\|v\|$, and $\left\|\left(T_{a_{n}}^{-1}-\widetilde{T}_{a_{n}}^{-1}\right) \widetilde{T}\right\|=\left\|T_{a_{n}}^{-1}\left(\widetilde{T}_{a_{n}}-T_{a_{n}}\right) \widetilde{T}_{a_{n}}^{-1} \widetilde{T}\right\| \leq \frac{2 M_{1} M_{2} g_{n}}{a_{n}}:=\frac{C_{1} g_{n}}{a_{n}}$. Let $c_{0}:=\frac{M_{2}}{4}$. Then it follows from (24) that

$$
g_{n+1} \leq\left(1-h_{n}\right) g_{n}+\frac{c_{0} h_{n} g_{n}^{2}}{\sqrt{a_{n}}}+C_{1} h_{n}\|v\| g_{n}+h_{n} a_{n}\|v\| .
$$

Let us assume that $C_{1}\|v\| \leq \frac{1}{2}$. Then

$$
g_{n+1} \leq\left(1-\frac{h_{n}}{2}\right) g_{n}+\frac{c_{0} h_{n}}{\sqrt{a_{n}}} g_{n}^{2}+h_{n} a_{n}\|v\| .
$$

Choose $a_{n}=16 c_{0}^{2} g_{n}^{2}$, so that $\frac{c_{0} g_{n}}{\sqrt{a_{n}}}=\frac{1}{4}$, and get

$$
g_{n+1} \leq\left(1-\frac{h_{n}}{4}\right) g_{n}+16 c_{0}^{2} h_{n}\|v\| g_{n}^{2}, \quad g_{0}=\left\|u_{0}-y\right\| \leq R,
$$

where $R>0$ is defined in (3). Take $h_{n}=h \in(0,1)$ and choose $g_{0}:=m$, where

$$
m<\frac{q+\frac{h}{4}-1}{16 c_{0}^{2} h\|v\|}, \quad q \in(0,1), \quad 1>q>1-\frac{h}{4}>0 .
$$

Then Lemma 2 with $\gamma=1-\frac{h}{4}$ and $p=16 c_{0}^{2} h\|v\|$ implies

$$
\left\|u_{n}-y\right\| \leq g_{0} q^{n} \rightarrow 0 \text { as } n \rightarrow \infty \text {. }
$$

Theorem 2 is proved. 


\section{Stability of the solution}

Assume that $F(y)=f$, where the exact data $f$ are not known but the noisy data $f_{\delta}$ are given, $\left\|f_{\delta}-f\right\| \leq \delta$. Then the DSM yields a stable approximation of the solution $y$ if the stopping time $t_{\delta}$ is properly chosen. The DSM is similar to (9):

$$
\dot{u}_{\delta}(t)=-T_{a(t)}^{-1}\left[A^{*}\left(F\left(u_{\delta}(t)\right)-f_{\delta}\right)+a(t)\left(u_{\delta}(t)-u_{0}\right)\right], \quad u_{\delta}(0)=u_{0},
$$

Let

$$
w_{\delta}:=u_{\delta}(t)-y, \quad g_{\delta}(t):=\left\|w_{\delta}\right\| .
$$

As in the proof of Theorem 1 we derive the inequality similar to (14):

$$
\dot{g}_{\delta} \leq-\frac{g_{\delta}}{2}+\frac{c_{0} g_{\delta}^{2}}{\sqrt{a(t)}}+a(t)\|v\|+\frac{\delta}{2 \sqrt{a(t)}}, \quad c_{0}:=\frac{M_{2}}{4},
$$

and apply Lemma 1. Rather than to repeat the arguments, given in the proof of Theorem 1 , we will use the results obtained in this proof. The constant $c_{1}$ in Theorem 1 is now replaced by $c_{\delta}:=c_{1}+0.5 \delta a^{-1.5}\left(t_{\delta}\right)$. As in the proof of Theorem 1 one gets the estimate $\left\|u_{\delta}(t)-y\right\| \leq a^{0.5}(t) \lambda^{-1}, \quad t \in\left[0, t_{\delta}\right]$. Let us define the stopping time $t_{\delta}$ from the equation

$$
0.5 \delta a^{-1.5}\left(t_{\delta}\right)=c_{1}
$$

This equation has a unique solution $t_{\delta}$, because $a(t)$ is decaying monotonically. Clearly, $\lim _{\delta \rightarrow 0} t_{\delta}=\infty$. Since $\lim _{t \rightarrow \infty} a(t)=0$ and $\lambda$ in our argument does not depend on $t_{\delta}$, the estimate $\left\|u_{\delta}\left(t_{\delta}\right)-y\right\| \leq a^{0.5}\left(t_{\delta}\right) \lambda^{-1}$ shows that $\lim _{\delta \rightarrow 0}\left\|u_{\delta}\left(t_{\delta}\right)-y\right\|=0$. Thus, we have proved the following theorem.

Theorem 3. Let $u_{\delta}:=u_{\delta}\left(t_{\delta}\right)$, where $u_{\delta}(t)$ solves problem (27) and $t_{\delta}$ is chosen in (28). Then $\lim _{\delta \rightarrow 0}\left\|u_{\delta}-y\right\|=0$.

\section{Stability of the iterative solution}

Assume that the equation is $F(u)=f, f$ is unknown, but the "noisy datum" $f_{\delta}$ is known, such that $\left\|f_{\delta}-f\right\| \leq \delta$. Consider the iterative process similar to (10):

$$
v_{n+1}=v_{n}-h_{n} T_{a_{n}}^{-1}\left[A^{*}\left(v_{n}\right)\left(F\left(v_{n}\right)-f_{\delta}\right)+a_{n}\left(v_{n}-u_{0}\right)\right], \quad v_{0}=u_{0},
$$

Let

$$
w_{n}:=v_{n}-y, \quad\left\|w_{n}\right\|:=\psi_{n},
$$

and choose $h_{n}=h$ independent of $n, h \in(0,1)$. A positive lower bound on $h$ is imposed in formula (35) below. An inequality similar to (25) takes the form:

$$
\psi_{n+1} \leq \gamma \psi_{n}+p \psi_{n}^{2}+\frac{h \delta}{2 \sqrt{a_{n}}}, \quad \psi_{0}=\left\|u_{0}-y\right\|,
$$


where

$$
\gamma:=1-\frac{h}{4}, \quad p:=16 c_{0}^{2}\|v\|, \quad a_{n}=16 c_{0}^{2} \psi_{n}^{2} .
$$

We stop iterations in formula (29) when $n=n(\delta)$, where $n(\delta)$ is the largest integer for which the inequality

$$
\frac{h \delta}{2 \sqrt{a_{n}}} \leq \kappa \gamma \psi_{n}, \quad \kappa \in\left(0, \frac{1}{3}\right),
$$

holds. One can use formula (31) for $a_{n}$ and rewrite this inequality as

$$
\frac{h \delta}{8 c_{0} \kappa \gamma} \leq \psi_{n}^{2}, \quad \kappa \in\left(0, \frac{1}{3}\right) .
$$

If (32) holds, then (30) implies:

$$
\psi_{n+1} \leq(1+\kappa) \gamma \psi_{n}+p \psi_{n}^{2}, \quad(1+\kappa) \gamma<1,
$$

and the conditions

$$
\gamma=1-\frac{h}{4}, \quad 0<\kappa<\frac{1}{3}, \quad h \in\left(\frac{4 \kappa}{1+\kappa}, 1\right),
$$

imply that $(1+\kappa) \gamma<1$ and $\frac{4 \kappa}{1+\kappa}<1$. If

$$
\psi_{0}<\frac{q-(1+\kappa) \gamma}{p}, \quad \text { where }(1+\kappa) \gamma<q<1, \quad \gamma=1-\frac{h}{4},
$$

then inequality (34) and Lemma 2 imply

$$
\psi_{n} \leq \psi_{0} q^{n},
$$

provided that

$$
n<n(\delta), \quad(1+\kappa) \gamma<q<1, \quad 0<\kappa<\frac{1}{3},
$$

where $n(\delta)$ is the largest integer for which inequality (32) holds. Clearly, $\lim _{\delta \rightarrow 0} n(\delta)=\infty$. Thus

$$
\lim _{\delta \rightarrow 0} \psi_{n(\delta)}=0 .
$$

We have proved the following result.

Theorem 4. Let the assumptions of Theorem 1 hold and $(1+\kappa) \gamma<1$. Assume that conditions (35) and (36) hold, and $\psi_{n}=\left\|v_{n}-y\right\|$, where $v_{n}$ is defined by equation (29). Then relations (37) and (39) hold, and $\lim _{\delta \rightarrow 0}\left\|v_{n(\delta)}-y\right\|=0$.

\section{References}

[1] Gavurin, M., Nonlinear functional equations and continuous analogues of iterative methods, Izv. Vuzov. Ser. Matematika, 5, (1958), 18-31. 
[2] Ortega, J., Rheinboldt, W., Iterative solution of nonlinear equations in several variables, SIAM, Philadelphia, 2000.

[3] Ramm, A. G. , Inverse problems, Springer, New York, 2005.

[4] Ramm, A. G. , Dynamical systems method for solving operator equations, Elsevier, Amsterdam, 2007.

[5] Ramm, A. G. , Hoang, N.S., Dynamical Systems Method and Applications. Theoretical Developments and Numerical Examples, Wiley, Hoboken, 2012

[6] Ramm, A. G. , Dynamical Systems Method (DSM) for general nonlinear equations, Nonlinear Analysis: Theory, Methods and Appl., 69, N7, (2008), 1934-1940.

[7] Ramm, A. G. , Stability of solutions to some evolution problems, Chaotic Modeling and Simulation (CMSIM), 1, (2011), 17-27.

[8] Ramm, A. G. , How large is the class of operator equations solvable by a DSM Newton-type method? Appl. Math. Lett, 24, N6, (2011), 860-865. 